\title{
Quand Ça Fait Si Mal: Protestation, Subversion et Espérance Dans le Psaume 137
}

\author{
Yacouba Sanon (Universite de L'Alliance Chretienne \\ D'ABIDJAN)
}

\begin{abstract}
This article examines Psalm 137 in the light of reggae songs by two Ivorian Rastas. The main objective is to demonstrate that the Psalm participates in the construction of a subversive identity by insisting on the transformative power of lament and collective memory. The article is arranged into three main sections: (1) the socio-historical context of the birth of Psalm 137; (2) the literary and canonical context; and (3) socio-theological and comparative analysis. The comparative reading of the different texts makes it possible to highlight the subversive and protest character of Psalm 137. It also makes it possible to highlight the need for reflection on the past, the value of decision making in the present and the need to desire a future built on justice. *
\end{abstract}

\section{RÉSUMÉ}

Cet article examine le Psaume 137 à la lumière de chansons reggae de deux rastas ivoiriens. L'objectif principal est de démontrer que ce Psaume participe de la construction d'une identité subversive en s'appuyant sur le pouvoir transformateur de la lamentation et de la mémoire collective. L'article s'articule autour de trois principaux axes: (1) le contexte socio-historique de la naissance du Psaume 137; (2) le contexte littéraire et canonique; et (3) l'analyse sociothéologique et comparative. La lecture comparative des différents textes permet de mettre en relief le caractère subversif et revendicatif du Psaume 137. Elle permet également de mettre en exergue le besoin réflexion sur le passé, la valeur des prises de décisions dans le présent et la nécessité d'aspirer à un avenir plus juste.

KEYWORDS: Psalm 137, Alpha Blondy, Tiken Jah Fakoly, Babylon, Zion, Protest, Subversion, Justice, Hope, Rasta, Reggae

\footnotetext{
Submitted: 05/05/2020; peer-reviewed: 21/08/2020; accepted: 25/08/2020. Yacouba Sanon, "Quand Ça Fait Si Mal: Protestation, Subversion et Espérance Dans le Psaume 137," Old Testament Essays 33 no. 3 (2020): 596-619. DOI: https://doi.org/10.17159/2312-3621/2020/v33n3a13.
} 


\section{A INTRODUCTION}

Le Psaume 137 a reçu l'attention de nombreux chercheurs en raison de son caractère à la fois attractif et répulsif. Certains le considèrent comme un chef d'œuvre poétique, ${ }^{2}$ l'un des plus beaux poèmes du psautier qui se termine malheureusement par "une imprécation monstrueuse." 3 Pour d'autres, c'est un Psaume déroutant mais en même temps important ${ }^{4}$ ou encore comme "le plus troublant de tous les psaumes du Psautier." ${ }^{5}$ Le Psaume 137 est complexe et reste insoumis aux efforts des spécialistes de l'enfermer dans leurs belles prisons académiques. ${ }^{6}$ Dans le but de comprendre ce Psaume plutôt énigmatique, différentes approches lui ont été appliquées. Des interprétations juives aux approches chrétiennes, de l'allégorie des Pères de l'église aux interprétations jugées culturelles, aucun effort n'est de trop pour chercher à comprendre le message laissé par le poète. Le présent travail s'inscrit dans cette dynamique d'interprétation du Psaume 137. Dans cet article, nous prenons des textes de

2 Arthur Weiser, The Psalms (The Old Testament Library; Trans. Herbert Hartl; Philadelphia: The Westminster Press, 1962), 794.

3 Samuel Terrien, The Psalms: Strophic Structure and Theological Commentary. (Grand Rapids: WBEPC, 2003), 865.

4 Frank L. Hossfeld and Erich Zenger, Psalms 3: A Commentary on Psalms 101-150 (Trans. Linda M. Maloney; Minneapolis: Fortress Press, 2011), 137.

5 Nancy deClaissé-Walford et al., The Book of Psalms (NICOT; Traduction libre de l'anglais, Y.S.; Grand Rapids: WBEPC, 2014), 953.

6 Il convient de mentionner brièvement quelques éléments concernant le Gattung, c'est-à-dire, le genre littéraire du Psaume. Allen tout comme Anderson reconnaissent que le Psaume 137 ne se prête pas facilement à la classification critique classique de la Critique des Formes. De l'avis de Allen, le "Psaume 137 est une version modifiée du chant de Sion." Leslie C. Allen, Psalms 101-150 (WBC 21, Waco: Word Books, 1983), 239; Anderson de son côté y voit trois tendances: une lamentation communautaire (vv. 1-4), un chant de Sion (vv. 5-6) et une imprécation (vv. 7-9); Arnold A. Anderson. The Book of Psalms: Vol II: Psalms 73-150 (NCB Commentary; Grand Rapids: WBEPC, 1972), 896. Dahood le caractérise tout simplement comme une lamentation; Mitchell Dahood, Psalms 101-150: A New Translation with Introduction and Commentary (AB; Garden City, NY: Doubleday, 1970), 269; deClaissé-Walford estime que dans la forme, le Psaume est une lamentation communautaire tandis que dans le fond il correspond à un Psaume d'imprécation; N. deClaissé-Walford et al., The Book of Psalms, 953. La structure du Psaume est également diversement appréciée. On retiendra ici les deux principales tendances. La première divise le Psaume en trois parties 1) vv. 1-4;2) vv. $5-6 ; 3$ vv. 7-9. La deuxième tendance propose également un découpage en trois parties, mais à une jonction différente; 1) vv.1-3; 2) vv. 4-6; 3) vv. 7-9. La question est donc de savoir si le verset 4 conclut la première section ou si elle introduit la deuxième section. Quoique certains auteurs avancent l'hypothèse d'un Psaume composite d'un point de vue critique, la tendance générale est de considérer le Psaume comme une unité littéraire cohérente. 
chants de deux rastamen ivoiriens, Alpha Blondy ${ }^{7}$ et Tiken Jah Fakoly ${ }^{8}$ comme grille de lecture des préoccupations de la jeunesse ouest africaine, mais aussi comme lunettes pour comprendre et interpréter le Psaume 137. Nous abordons donc le texte avec une herméneutique qui affirme l'autorité de l'Écriture tout en privilégiant le vécu et l'expérience quotidienne des Africains. Du point de vue anthropologique, cette double perspective 'émique' et 'étique' permet d'une part d'examiner le langage et la structure interne du Psaume pour déterminer le point de vue de son auteur et, d'autre part, d'analyser le Psaume d'une manière qui transcende le cadre culturel des israélites pour prendre en compte celui du lecteur moderne.

L'objectif principal est de démontrer que ce Psaume participe de la construction d'une identité subversive en s'appuyant sur le pouvoir transformateur de la lamentation et de la mémoire collective. L'approche ainsi préconisée espère prendre en compte les trois contextes primordiaux que sont, celui de l'auteur et des premières audiences, celui du texte et des cotextes, puis enfin celui des lecteurs contemporains. L'étude s'articulera autour des axes suivants: (1) le contexte socio-historique de la naissance du Psaume 137; (2) le contexte littéraire et canonique; (3) l'analyse socio-théologique et comparative. Mais auparavant, un état de la recherche sur le Psaume 137 s'impose.

\section{B ÉTAT DES RECHERCHES SUR LE PSAUME 137}

Le Psaume 137 est un lieu de la mémoire traumatisée. Il articule d'une manière poignante le sentiment de fragilité d'un groupe de déportés, contrôlés et dominés par des maîtres sarcastiques et cruels. Les intérêts des commentateurs classiques se sont focalisés sur les questions liées à l'origine et à la date de composition du Psaume tandis que des auteurs plus récents se sont intéressés plus orientés vers les questions d'ordre socio-politiques dont regorge ce Psaume.

Les commentateurs s'accordent pour reconnaitre que le Psaume renvoie à l'exil des juifs en Babylonie au VIème siècle et à leur expérience quotidienne durant la captivité. Cependant, les divergences concernant sa date de composition et son origine semblent irréductibles. Sur cette question les opinions sont divergentes. Les évènements évoquent certes le souvenir des vagues successives de déportations des israélites en Babylonie par les Chaldéens en 597 puis en 587 mais la date de composition reste une pomme de discorde. Certains auteurs pensent que le Psaume a été composé pendant l'exil babylonien; c'est le

7 Alpha Blondy, de son vrai nom Seydou Koné, est né en 1953 à Dimbokro en Côte d'Ivoire. C'est un chanteur de reggae dont la renommée est d'envergure internationale. Il a à son actif d'innombrables albums.

8 Tiken Jah Fakoly, de son vrai nom Moussa Fakoly Doumbia est né le 23 juin 1968 à Odienné en Côte d'Ivoire. Il est également artiste musicien chanteur de reggae. 
cas de Briggs qui estime que la destruction de Jérusalem est encore fraîche dans la mémoire du psalmiste dont le ressentiment envers les Édomites est toujours vif. Terrien également croit que le Psaume aurait été composé dans la première partie du VIème siècle par un auteur qui serait un témoin des évènements qu'il raconte ou qui appartiendrait à la seconde génération des déportés.

D'auteurs pensent que le Psaume a été composé à Jérusalem après le retour des israélites de la captivité. Oesterley affirme que le Psaume a été composé en 538 comme une sorte de mémoires par un ancien exilé. Pour Weiser, la vue des décombres de la ville aux lendemains du retour est à l'origine de la composition du Psaume; Dahood, suivi par Allen, estime que le Psaume a été composé peu après le retour des exilés; Anderson affirme quant à lui que l'auteur qui aurait été un témoin oculaire de la chute de Jérusalem, aurait été lui-même déporté, n'a composé le Psaume qu'après son retour de l'exil mais avant la reconstruction du second temple. Récemment, Hossfeld et Zenger ont pris le contre-pied de la plupart de leurs prédécesseurs en suggérant que le Psaume 137 a été composé beaucoup plus tardivement à Jérusalem (accents sur le temple et la musique du temple) par des musiciens qui appartiendraient au groupe des asaphites. ${ }^{9}$

Bien que le Psaume 137 soit apparu dans un espace particulier et à un temps spécifique, l'expérience qu'il relate est familière à celle vécue par d'autres groupes sociaux, d'où sa facilité d'adaptation en dehors des cercles juifs. En particulier, le Psaume 137 fait écho à l'expérience ancienne et récente des peuples africains au Sud du Sahara, en particulier la traite des noirs et la colonisation. ${ }^{10}$ En Amérique du Nord, le Psaume 137 a pendant longtemps été une source d'inspiration dans les mouvements de revendication sociales et de

9 Voir Charles A. Briggs and Emilie G. Briggs, The Book of Psalms (vol. II; CEC; Edinburgh: T \& T Clark, 1907), 485; S. Terrien, The Psalms: Strophic Structure and Theological Commentary (Grand Rapids: WBEPC, 2003), 867; W.O.E. Oesterly, The Psalms (London: SPCK, 1955), 545-546; A. Weiser, The Psalms, 794; Mitchell Dahood, Psalms 101-150: A New Translation with Introduction and Commentary (AB Garden City, NY: Doubleday, 1970), 269; Leslie C. Allen, Psalms 101-150. (WBC 21; Waco: Word Books, 1983), 239; Arnold A. Anderson, The Book of Psalms. Vol II. Psalms 73-150 (NCBC; Grand Rapids: WBEPC, 1972), 897; F. L. Hossfeld and E. Zenger, Psalms 3: A Commentary on Psalms 101-150 (Minneapolis: Fortress Press, 2011), 514.

10 L'expression Afrique subsaharienne désigne un espace géographique vaste et très varié qui se situe en dessous du Sahara, cette étendue désertique qui couvre toute la partie Nord du continent africain, d'Ouest en Est. Cependant, l'espace géographique et culturel auquel cette étude se réfère aux pays francophones du Golfe de Guinée-Benin, Togo, Côte d'Ivoire, Guinée, Sénégal et leurs voisins du Nord, Mali, Burkina et Niger. Ils ont en commun un passé colonial similaire, la langue française, une monnaie commune, une croissance économique globalement très faible et un taux de paupérisation de la population en général très élevé. 
lutte contre le racisme. ${ }^{11}$ Sadler soutient que le Psaume 137 était un chant subversif, notamment dans son dernier acte. À ce propos il écrit: "Le chant dans les versets $7-9 \ldots$ est un loup imprécatoire vêtu de peaux de mouton d'un chant du temple de Jérusalem chanté par un poète de Juda plié mais non cassé devant ses ravisseurs." ${ }^{12}$ Dans le même sens, Bridgeman propose une lecture du Psaume 137 à la lumière des expériences récentes des Africains Américains et postule que ce Psaume est une protestation contre les dégradations et les humiliations, un refus de capituler devant la brutalité humaine. ${ }^{13}$

Des études plus récentes sur le Psaume 137 soulignent la diversité des approches mises en œuvre pour en saisir la signification pour les croyants de notre temps. Simango par exemple fait une analyse "tridimensionnelle" basée sur l'organisation interne du psaume, les questions relatives aux contextes historiques et littéraires, et le contexte canonique. ${ }^{14}$ Le mérite de cette étude réside dans la rigueur observée par l'auteur en vue de parvenir à une compréhension globale du psaume. Avec cette démarche exégétique classique, l'auteur aboutit à une conclusion certes 'objective' mais qui souffre d'un manque d'engagement critique avec les structures d'oppression. Le message du Psaume 137, ce n'est pas seulement un refus de s'associer au cirque des blasphémateurs de Yahvé, ce n'est pas seulement la volonté de demeurer fidèle à Yahvé en comptant sur sa souveraineté. Le Psaume 137 est aussi une révolte contre la machine oppressive qu'était l'empire babylonien et, par extension, une dénonciation de tout système d'oppression où qu'il se trouve.

L'analyse de Ramantswana du Psaume 137 se rapproche de cette perspective et de celle adoptée dans le présent travail. ${ }^{15}$ Partant de l'expérience des chants de lutte en Afrique du Sud, Ramantswana fait une lecture "décoloniale" du Psaume 137 et estime que ce psaume est "une chanson de lutte" épique. Les chants de lutte, explique-t-il, sont des compositions politiquement motivées qui exposent les faits et méfaits politiques et interpellent les acteurs de la scène politique. ${ }^{16}$ Selon Ramantswana, le Psaume 137 serait en fait une "anthologie" dont l'origine remonte à la période exilique. Ainsi, les trois strophes

11 David W. Stowe, "History, Memory, and Forgetting in Psalm 137," The Bible in the Public Square 27 (ed. M. E. Chancey, C. Meyers and C. E. Meyers; Atlanta: SBL Press: 2014), 138.

12 Rodney S. Sadler Jr., "Singing a Subversive Song: Psalm 137 and 'Coloured Pompey," The Oxford Handbook of Psalms (ed. W. P. Brown; Traduction libre de l'anglais, Y.S.; Oxford: Oxford University Press, 2014), 453.

13 Valerie Bridgeman, "A Long Way from Home': Displacement, Lament, and Singing Protest in Psalm 137," Perspectives in Religious Studies 44/2 (2017): 213-223. 14 Daniel Simango, “A Comprehensive Reading of Psalm 137," OTE 31 (2018): 217242.

15 Hulisani Ramantswana, "Song(s) of Struggle: A Decolonial Reading of Psalm 137 in Light of South Africa's Struggle Songs," OTE 32 (2029): 464-490.

16 Ibid., 466-467. 
de notre Psaume correspondraient à trois différents chants de lutte qui ont été compilé vraisemblablement, selon l'auteur, après le retour de l'exil-vv.1-4: chant de protestation; vv. 5-6: chant de désolation; et vv.7-9: chant de vengeance ou de guerre. ${ }^{17}$ Les chants de lutte ou de libération, rappelle Ramantswana, ne connaissent pas de frontière. Ils s'adaptent facilement aux nouvelles situations, meurent, renaissent et se font une autre vie. ${ }^{18}$ Selon Ramantswana, ce serait le cas du Psaume 137, qui aurait retrouvé une seconde vie après le retour de l'exil lorsque la communauté juive est passée de la domination babylonienne sur un sol étranger, à la domination perse sur ses terres ancestrales.

\section{TRADUCTION DU PSAUME 137}

1. Là-bas, au bord des fleuves de Babylon

Nous étions assis et nous pleurions en nous souvenant de Sion

2. Sur les peupliers/saules au milieu d'elle,

Nous avions suspendu nos harpes/cithares

3. Car là, nos tortionnaires/bourreaux

Nous demandèrent des chansons,

Et nos ravisseurs des chants joyeux:

"Chantez-nous le chant de Sion!"

4. Comment pouvons-nous chanter le chant de YHWH sur une terre étrangère?

5. Si je t'oublie, Jérusalem,

Que ma droite oublie.

6. Que ma langue colle à mon palais

Si je ne me souviens de toi,

Si je n'élève pas Jérusalem en tête de ma joie

7. Seigneur, souviens-toi des Édomites,

Eux qui disaient au jour de Jérusalem: "rasez, rasez jusqu'à ses fondations!"

8. Fille de Babylone, la dévastée,

Heureux qui te traitera comme tu nous as traités;

9. Heureux qui saisira tes nourrissons et les fracassera sur le roc.

\section{Contexte socio-historique: Naissance d'un chant de lamentation}

Le Psaume 137 a vu le jour dans un contexte de douleur et de tourments. L'évaluation de Weiser est pertinente:

[Le Psaume 137] révèle les souffrances et les sentiments de personnes qui ont peut-être vécu de première main les jours douloureux de la conquête et de la destruction de Jérusalem en 587 avant JC, qui ont partagé le fardeau de la captivité babylonienne et

17 Ibid., 469, 472-483.

18 Ibid., 383-384. 
après leur retour dans leur patrie maintenant, à la vue de la ville encore en ruine, donnent libre cours avec une intensité passionnée aux sentiments qui sommeillent dans leurs cœurs. ${ }^{19}$

C'est avec une honnêteté déconcertante que le psalmiste a composé ce poème. Il ne laisse aucun doute quant à l'identité des acteurs concernés dans la tragédie dont il se fait le porte-parole. Ici, apparait une faiblesse dans l'évaluation de Weiser et d'autres qui soutiennent que le Psaume 137 a été écrit par un témoin ayant lui-même vécu les évènements décrits. Si être témoin oculaire est un avantage, ce n'est pas une condition sine qua non pour composer un poème. Zenger estime plutôt que l'auteur n'avait nullement besoin d'être contemporain des faits. Pour lui, le Psaume a été composé à Jérusalem non pas comme un chant de résistance, mais plutôt comme un poème rétrospectif sur la situation exilique et postexilique. ${ }^{20}$ Quoique la suggestion de Zenger ait beaucoup de mérite, il reste que ce Psaume présuppose et exprime une réalité, l'angoisse, la colère et la révolte (intérieure) devant l'horreur et l'atrocité. Les lamentations ne naissent pas simplement à partir d'un calcul froid en vue de justifier une position ou une attitude ou pour diffuser des préjugés. Le livre des Lamentations prouve cela suffisamment et souligne également le lien entre lamentation et poésie déjà évident dans les Psaumes. Commentant cette relation, Katongole a fait remarquer le lien entre la douleur et la parole. La douleur, dit-il, "détruit la capacité de parole des victimes" tandis que "la poésie est une tentative de donner des mots à ce qui est trop profond pour être dit, de dire l'indicible." ${ }^{21}$ La violence et l'oppression engendrent des destructions et des pertes profondes auxquelles la lamentation cherche à donner une voix. "Les sentiments indiquent une perte bien plus profonde: la perte d'un ancrage pour l'avenir, la perte de la communauté, et, finalement, la perte de l'humanité." 22

Les chants ou poèmes de lamentations se rattachent à des événements douloureux. Ils émanent du désir de rendre publique les sentiments qui rongent leurs auteurs et qu'ils ne peuvent contenir. Comme la discussion sur l'auteur du Psaume 137 l'atteste, le fait d'avoir vécu soi-même des événements dramatiques ne devrait pas être retenu comme condition sine qua non pour être en mesure de composer une élégie. Ce critère qualifie certes, mais il ne disqualifie pas. Les témoins oculaires passent souvent leurs souvenirs à la génération suivante et

${ }^{19}$ Weiser, 794. Westminster John Knox, 1996), 514.

21 Emmanuel Katongole, Born from Lament: The Theology and Politics of Hope in Africa (Traduction libre de l'anglais, Y.S.; Grand Rapids: WBEPC, 2017), 42.

22 Katongole, Born of Lament, 62. Katongole insiste particulièrement des poésies et des chants, des poésies et de l'art inspirées des décennies de violences et d'atrocités en Afrique centrale, notamment dans son pays l'Ouganda et à l'Est du Congo, Ibid., 62100 [Traduction libre de l'anglais, Y.S.]. 
l'étude de l'histoire permet de se plonger dans le passé des peuples. En prenant en compte ces perspectives, il est possible et même utile d'examiner le Psaume 137 à la lumière de chants tirés du patrimoine musical contemporain de la Côte d'Ivoire. Ces chants ont des parallèles avec le Psaume 137. Je mentionnerai ces parallèles à mesure que nous progressons dans l'analyse du Psaume 137 axée sur la division tripartite du texte et qui s'articule autour de trois temps que sont la réflexion, la résolution et l'action.

\section{CONTEXTE LITTERAIRE ET CANONIQUE}

\section{Réflexions sur le Passé}

La première strophe du Psaume 137 (vv. 1-4) est un voyage dans le passé. La voix qui s'exprime ici évoque le souvenir mélancolique d'une expérience communautaire qui a profondément marqué les esprits. Avec un peu d'exagération, nous pouvons affirmer que cette strophe "reste à toujours l'expression la plus parfaite de la douleur patriotique et de la dignité dans le malheur."23

Les deux premiers versets renvoient au site de l'expérience avec des

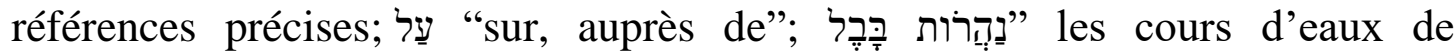

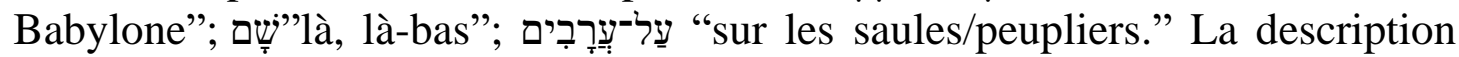
topographique est précise et elle a pour but de marquer le contraste saisissant avec l'objet du souvenir que le psalmiste évoque dans le même verset. C'est précisément à Babylone que les exilés se souviennent de צִּיון "Sion."24 D’une manière ironique, l'éloignement géographique a créé le rapprochement psychologique et émotionnel. Le souvenir de Sion provoque des pleurs. Le psalmiste ne dit pas en quoi consiste ces souvenirs, mes ses larmes témoignent de ses sentiments. Lelièvre remarque avec justesse que pendant l'exil, les israélites avaient certainement eu le droit de se rassembler, des rassemblements qui selon lui seraient "culte en souvenir de Sion où l'on célébrait un culte en l'honneur de Dieu." 25 Toujours selon Lelièvre, c'est l'impossibilité de rendre un culte à YHWH qui est la cause de leur chagrin. "Ils pleurent la ville qu'ils ont perdue, le temple dont ils se sont séparés, la liturgie qu'ils ne peuvent pas célébrer et les sacrifices qu'ils ne peuvent plus offrir." ${ }^{26}$ La posture des exilés, assis à même le sol et pleurant, est une manifestation extérieure de deuil bien

${ }^{23}$ Frédéric Gaudet, La Bible annotée: A.T. 6 Psaumes (Saint-Légier: PERLE, 1982), 354.

24 George Savran, "How Can We Sing a Song of the Lord? The Strategy of Lament in Psalm 137" (ZAW 112; ed. H. C. Schmitt and G. Wanke; Berlin: Walter de Gruyter, 2000), 44-45.

25 A. Lelièvre et A. Maillot, Les Psaumes: Chants d'amour 76-150 (Lyon: Olivetant, 2008), 398.

26 Lelièvre et Maillot, Les Psaumes, 398. 
connu en Afrique. En somme, les exilés pleurent parce qu'ils ont perdu les marques essentielles de leur identité. ${ }^{27}$

La suspension des harpes est le signe que le temps n'est pas à la célébration, mais bien à la lamentation. On pourrait voir dans cet acte une protestation contre l'oppression et la brutalité. C'est d'ailleurs de cette manière que Bridgeman lit ces versets, à la manière des sit-in organisés par les mouvements de revendication sociale. ${ }^{28}$ Le problème ici est que, les exilés ne semblent pas être en train de revendiquer quelque chose. Dans les deux premiers versets, leur mouvement est orienté non pas vers l'extérieur, mais bien vers l'intérieur, à moins que l'on ne prenne la préposition 'כ̣ comme motivant le geste des exilés. Cela reviendrait à dissocier le rapport entre le fait de s'asseoir et de pleurer d'avec le fait de suspendre les harpes. Cela reviendrait également à atténuer l'expression רְְְִָּרָרנוּ qui est centrale dans cette partie de la strophe.

Les versets 3-4 renforcent l'interprétation précédente selon laquelle le rassemblement des exilés avait une visée introspective plutôt que revendicative. Selon Becking, des études récentes montrent que les exilés jouissaient de conditions matérielles plutôt bonnes. ${ }^{29}$ Leur lamentation ne portait donc pas premièrement sur leur bien-être physique, mais sur leur détresse intérieure, une situation exacerbée par la provocation de leurs maîtres. La requête de leurs tortionnaires est perçue par plusieurs comme moquerie ou une plaisanterie de mauvais goût. Elle accentue leur sentiment d'aliénation, car allant à l'encontre de leurs convictions religieuses et de leur zèle patriotique. L'existence du genre "chants de Sion" n'est pas établie avec certitude dans les Psaumes. Cependant, des Psaumes tels que Pss 46, 48, 122, 126, etcetera, qui célèbrent la grandeur de YHWH en Sion ainsi que "l'imprenabilité" et l'inviolabilité de Sion et qui prédisent le malheur et la chute de ceux qui l'attaquent pourraient être considérés comme Psaumes de Sion. La réponse des exilés sous forme de question "Comment pouvons-nous chanter un chant de YHWH sur une terre étrangère?" révèle leur dilemme. D'une part, ils font face à sa leur conscience qui luer interdit de chanter, car ce serait synonyme d'oublier Jérusalem. D'autre part, ils font face à l'oppresseur à qui ils doivent obéir.

Selon Savran, la réponse du psalmiste consiste précisément aux paroles des versets 5-6. Pour lui, le psalmiste répond à sa propre question "en ne chantant pas au pluriel, en ne mentionnant pas Dieu, et en déclarant le souvenir de Jérusalem comme quelque chose au-delà de la joie." 30 Abondant dans le même

27 John Ahn, “Psalm 137: Complex Communal Laments," JBL 127 (2008) :279.

28 Bridgeman, "A Long Way from Home," 217-218.

29 Bob Becking, "Memory and Forgetting in and on Exile: Remarks on Psalm 137," Remembering and Forgetting in Early Second Temple Judah (ed. E. Ben Zvi and Christoph Levin; Tübingen: Mohr Siebeck, 2012), 294.

30 Savran, "How Can We Sing...?" 53 [Traduction libre de l'anglais, Y.S.]. 
sens, Sadler estime également que le psalmiste n'avait d'autre choix que de chanter; "il devait chanter pour survivre" et le chant en question est voilé dans les versets 7-9. ${ }^{31}$ Le psalmiste chante donc un prétendu chant de Sion qui est en fait un acte de résistance. Contrairement aux deux précédents auteurs, Zenger affirme que la réponse du psalmiste à la requête des conquérants est un refus catégorique de chanter. ${ }^{32}$ En tout état de cause, le psalmiste semble avoir retrouvé sa voix. Ses réflexions ne sont pas restées stériles. Après maintes hésitations et luttes intérieures, sa décision est prise. Chanter le chant de Sion, ou le chant de YHWH sur un sol étranger est tout simplement hors de question.

En somme, le premier couplet du Psaume 137 est un appel à se souvenir des jours passés. Le psalmiste réussit son pari en faisant revivre ce passé à travers un personnage représentant les exilés. Cette évocation d'une mémoire collective a un pouvoir fédérateur dans le sens où elle réunit les générations passées et présentes autour d'une identité religieuse et nationale. Cette dimension idéologique est renforcée si l'on admet que le Psaume a été composé après l'exil. Le Psaume devient un appel à chaque nouvelle génération à adopter le point de vue développé et à emboiter les pas des prédécesseurs. ${ }^{33}$

Comme la première strophe du Psaume 137, le chant de l'artiste ivoirien Tiken Jah Fakoli, "Days of Slavery" est une réflexion sur le passé colonial de l'Afrique plusieurs générations après les événements.

Days of slavery ${ }^{34}$

Do you remember the days of slav'ry? (6x)

And how they beat us... (do you remember the days of slav'ry?)

And how they worked us so hard.

(Do you remember the days of slav'ry?)

And how they used us... (do you remember the days of slav'ry?)

Till they refuse us... (do you remember the days of slav'ry?)

Do you remember the days of slav'ry?

(Do you remember the days of slav'ry?)

Mm... And a big fat bull.

Mm... We usually pull it ev'rywhere.

Mm... We must pull it.

Mm... With shackles around our necks.

31 Sadler, "Singing a Subversive Song," 452.

32 Zenger, 515. Rappelons que pour Zenger, le Psaume a été compose bien plus tard à Jérusalem par les Asaphites dans l'optique de défendre l'attitude des membres de leur groupe pendant l'exil.

33 Becking, "Memory, Forgetting and Exile," 299.

34 Tiken Jah Fakoly, "Days of Slavery," Racines, Universal Music Division Barclay, 2015. 
Mm... And I can see it all no more.

Do you remember the days of slav'ry?

(Do you remember the days of slav'ry?)

My brother feels it... (do you remember the days of slav'ry?)

Including my sisters too... (do you remember the days of slav'ry?)

Some of us survive... (do you remember the days of slav'ry?)

Showing them that we are still alive.

(Do you remember the days of slav'ry?)

Do you remember the days of slav'ry?

Oh slav'ry day (do you remember the days of slav'ry?)

Oh slav'ry day (do you remember the days of slav'ry?)

Cry and remember, please remember.

(Do you remember the days of slav'ry?)

Ce chant est une lamentation de type communautaire dans laquelle l'auteur s'identifie avec les souffrances de ses ancêtres soumis aux travaux forcés et torturés par les colons. On remarquera dans ce chant l'intégration entre le "nous" communautaire et le "je" individuel. Cette transition entre le pluriel et le singulier, entre la communauté et l'individu ne crée aucune tension dans ce texte. A notre avis, le même phénomène est observable dans la deuxième strophe du Psaume 137 où le psalmiste passe du pluriel au singulier. Ce changement sans avertissement semble déranger certains auteurs qui voient en cela une diversité d'origines dans le Psaume.

A la manière des psalmistes, l'auteur de "Days of Slavery" veut ne pas oublier le passé. D'où la répétition de la question languissante: "Do you remember the days of slavery?" On sent ici un souci chez l'auteur, le souci de conserver le passé pour mieux construire le présent et affronter l'avenir. Il ne s'agit pas de lui seul, mais de tous ses auditeurs: "Te souviens-tu des jours de l'esclavage?" Cela résonne comme une expression de la troisième strophe du Psaume 137, au v. 7 où l'auteur parle du "jour de Jérusalem," un euphémisme pour caractériser le siège, la prise et la destruction de Jérusalem par les babyloniens. Le chant du reggaeman n'est certes pas un chant religieux. C'est un chant militant, un plaidoyer pour la prise en compte de l'histoire douloureuse des noirs. A sa manière, l'artiste averti contre le danger de l'oubli. On peut regretter qu'il ne propose aucune solution, aucune réponse, aucun encouragement, mais il a fait sa part et cela ne lui sera pas enlevé. Le chant est déjà en lui-même un engagement.

Le Psaume 137, cependant, offre en sa deuxième strophe une ancre solide contre l'oubli et contre les angoisses de la mémoire douloureuse. 


\section{Résolutions pour les temps présents}

La deuxième strophe du Psaume 137 (vv.5-6) est une résolution ferme du psalmiste. On remarquera le passage de la première personne du pluriel "nous" dans la première strophe à la première personne du singulier "je" dans cette strophe qui est admirablement structurée sous forme chiastique. Le psalmiste

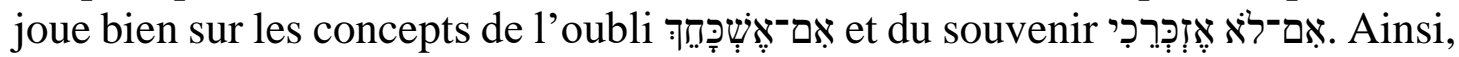
la deuxième strophe se rattache sémantiquement à la première et prépare en même temps la troisième. Dans une suite de propositions conditionnelles, le psalmiste fait le serment d'ériger Jérusalem au sommet de toute joie. L'imprécation du psalmiste contre lui-même est diversement interprétée par les auteurs. Pour la plupart, les hypothèses de la droite qui oublie la langue qui se colle au palais se rapportent simplement à l'impossibilité du psalmiste d'exercer sa fonction s'il venait à oublier Jérusalem. En d'autres termes, le psalmiste cesserait toute activité liée au chant et au jeu des instruments de musique. Zenger affirme qu'il s'agit plutôt d'une métonymie qui désigne la vie entière du psalmiste. C'est comme si le psalmiste jurait par sa propre vie. ${ }^{35}$ Étant donné la structure et l'organisation de cette strophe, il est possible d'affirmer que la résolution du psalmiste est ferme et finale. Sa loyauté envers Jérusalem est irrévocable et non négociable. Ses geôliers peuvent restreindre ses mouvements, mais ils ne peuvent pas enfermer son imagination qui prend librement le chemin de retour vers les terres plus familières de ses ancêtres.

\section{$3 \quad$ Aspirations à un avenir plus juste}

Dans cette strophe (vv. 7-9), la prière du psalmiste prend un accent particulièrement violent. Le désir de vengeance n'est ni refoulé ni réprimé. Pourtant, la communauté n'a aucun moyen ni pour se défendre, ni pour riposter face aux oppresseurs. Dans cette relation oppresseur-opprimé, aucun recours en justice n'était envisageable. Par conséquent, il fallait en appeler à Dieu, d'où, le verset 7. Ici, le thème du "souvenir" revient encore, mais cette fois avec un

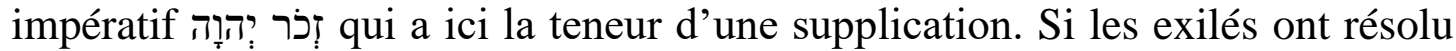
de ne pas oublier Jérusalem, alors YHWH doit lui aussi se souvenir de Jérusalem. Demander au Seigneur de se souvenir des Édomites, c'est lui demander d'agir en faveur de Jérusalem. C'est un appel à Dieu pour exercer la justice. La prière contre les Édomites n'est pas spécifique. Cela serait certainement dû aux prophéties de malheur qui les visaient (Jr 49.7-22; Ab 1-21). Puisqu'ils étaient déjà dans le collimateur de Dieu, un simple rappel est suffisant. Néanmoins, la prière contre eux au v.7 semble être motivée par un désir de vengeance. Le même désir de vengeance est observé à l'égard de Babylone. Le psalmiste est plus spécifique et plus virulent dans sa demande. Le degré de violence et de haine exprimé dans ces paroles est inouï et n'a d'égal qu'avec des paroles similaires.

\footnotetext{
${ }^{35}$ Zenger, "A God of Vengeance?” 518.
} 
Annonçant le jugement de Dieu sur la Samarie, Osée déclarait: "Samarie sera punie parce qu'elle s'est révoltée contre son Dieu. Ils tomberont par l'épée, leurs petits enfants seront écrasés et l'on fendra le ventre de leurs femmes enceintes." (Os 14.1) De son côté, Esaïe prophétisa contre Babylone en ces termes: "Tous ceux que l'on trouvera seront transpercés et tous ceux que l'on attrapera tomberont sous les coups de l'épée. Leurs enfants seront écrasés sous leurs yeux, leurs maisons seront pillées et leurs femmes seront violées." (Es 13.15-16). Ces paroles de l'Écriture sont choquantes. Pourtant, elles sont bien "parole de Dieu," (cf. Os 4.1) contre son peuple dans le premier cas, et "oracle," c'est-à-dire message divin, (cf. Es 13.1) dans le deuxième cas. Du reste, Calvin trouve dans ces passages de l'Écriture, le fondement qui autorise le psalmiste à prier comme il l'a fait. Pour lui, la prière du psalmiste vient simplement confirmer les prophéties antérieures. Avec le style qui le caractérise, il écrit:

Prier pour la vengeance aurait été injustifiable, si Dieu ne l'avait pas promis, et si le parti contre lequel il était recherché n'était pas réprouvé et incurable; car quant aux autres, même à nos plus grands ennemis, nous devrions souhaiter leur amendement et leur réforme. ${ }^{36}$

Puisque le psalmiste reprend à son compte les paroles de Dieu lui-même, c'est donc à Dieu seul que revient l'exécution de ses paroles. Le désir de se venger est-il pour autant "injustifié" comme le suggère Calvin? Si nous avons bien compris le Réformateur de Genève, un tel sentiment ne devrait pas animer le croyant dès lors qu'il s'agit des "autres." En d'autres termes, en dehors des Édomites, des Samaritains, et des Babyloniens, s'emporter dans la prière contre ses ennemis est à proscrire. Cet avis est certainement partagé par de nombreux croyants qui éprouvent une répulsion naturelle face à ce sentiment irréligieux. D'autres par contre voient dans la prière imprécatoire des vv. 7-9 du Psaume 137 un espace de liberté et d'émancipation par la parole. André Wénin est de ceux-là, lui qui affirme:

Dans la mesure où il reçoit un espace de confiance où se dire sans fard et sans honte, le désir de vengeance peut se décanter et laisser apparaître sa source: le sentiment d'être victime d'une injustice, d'une violence non méritée-un sentiment dont la force potentiellement ravageuse apparaît précisément dans la violence des paroles prononcées. ${ }^{37}$

Le Psaume se termine par un renversement de situation. Au début du Psaume, le portrait de Jérusalem est celui d'une ville défaite et dont les habitants sont dans la détresse en captivité. À la fin, l'image de Jérusalem qui se présente est celle

36 John Calvin, Commentary on the Book of Psalms 93-150 (Calvin's Commentaries vol. VI; Trans. J. Anderson; Grand Rapids: Baker Book House, 1979), 196.

37 André Wénin, Psaumes censurés: quand la prière a des accents violents (Lire la Bible 192, Paris: Cerf, 2017), 174. 
d'une ville qui renaîtra de ses cendres. Le psalmiste et les exilés, au départ vulnérables et malheureux retrouvent leur vraie identité en pleurant ensemble, en se lamentant ensemble et en priant ensemble. A travers leur lamentation, ils ont pu dire l'indicible. Avec leurs voix ils ont trouvé le chemin de la liberté et de l'espérance. Le Psaume 137 peut s'offrir alors comme une prière type. C'est la prière des pauvres, des opprimés, des victimes et de ceux qui souffrent, en particulier de ceux qui souffrent injustement. Elle n'a pas pour objet de faire des anciens opprimés de nouveaux oppresseurs. Non! Elle vise la déconstruction de l'oppression, de l'exploitation et de la domination. Le Psaume 137 est une prière qu'il convient alors d'adresser à Dieu "quand ça fait si mal."

\section{$4 \quad$ Le Psaume 137 et le Nouveau Testament}

La prière contre les ennemis dans le Psaume 137 atteint un degré de férocité et d'animosité pétrie d'une haine totale. Le peuple de Dieu demande à Dieu de le venger de ses ennemis en exerçant contre eux un jugement sans pitié. Cette haine viscérale contre autrui semble en tout point s'opposer à l'enseignement de Christ et du Nouveau Testament qui met l'accent sur l'amour et le pardon. Quel doit alors être l'attitude des chrétiens face au Psaume 137 et aux autres Psaumes d'exécration qui prônent la destruction et la haine des ennemis? Pour certains, la réponse est claire-les Psaumes d'imprécation d'Israël n'ont pas de place dans la piété chrétienne et doivent simplement être proscrits et de la liturgie et de la piété personnelle. Il s'agit donc d'ignorer ces portions de l'Écriture sainte qui mettent mal à l'aise notre sensibilité d'hommes et de femmes du $21^{\text {eme }}$ siècle. A côté des tenants de cette position extrême, il y a ceux qui esquivent le problème posé par le texte vis-à-vis de l'éthique chrétienne. A la question de savoir si le chrétien peut faire sien les paroles du psalmiste et les prier, ils répondent en mettant en garde ceux qui ont expérimenté l'horreur et la déshumanisation contre le danger de la vengeance. Les éditeurs de la Bible Africaine sont prudents lorsqu'ils écrivent qu' 'En suivant l'exemple de Jésus et son enseignement, les chrétiens doivent éviter toute sorte de vengeance." ${ }^{38}$ Le seigneur a certes vertement repris Jacques et Jean pour leur esprit belliqueux et revanchard contre un petit village Samaritain (Luc 9:52-56), mais il a aussi proclamé un jugement sévère contre Chorazin et Bethsaïda à cause de leur incrédulité (Matt 11:20-24). Le reproche du Seigneur nous indique une orientation sur l'utilisation qu'il ne faut pas faire des privilèges que Dieu nous donne: l'abus et le détournement d'usage.

Le Psaume 137 et les psaumes d'imprécation ne sont pas des armes à feu dont le croyant doit faire usage à volonté pour assouvir ses passions déréglées ou exercer une vengeance personnelle. Thomas Römer a raison lorsqu'il met en garde contre le danger de pervertir ce Psaume en le transformant "en un 
programme de condamnation ou de d'extermination de ses adversaires." 39 Ils constituent pour le chrétien un véhicule qui peut l'aider à canaliser sa colère, ses frustrations et sa haine dans une attitude de soumission à Dieu. ${ }^{40}$ Les chrétiens sont appelés à haïr le mal et à prier pour en être délivrés, "délivre-nous du mal." Le Psaume 137 nous aide à démasquer et dénoncer le mal dans toute sa laideur et dans toute sa gravité. Par ce Psaume, nous avons l'autorisation et le devoir de prier pour que les racines (les enfants) du mal, de la terreur et de l'oppression soient exterminées. C'est une prière radicale, et, peut-être une prière politique. C'est aussi le sens de la prière que notre Seigneur a enseignée à ses disciples: "Que ton règne vienne."

A l'instars de l'auteur du Psaume 137 et en alignement avec l'enseignement du Seigneur Jésus-Christ, des poètes-chantres se sont fait les porte-voix de la misère et des souffrances des masses exploitées et humiliées. ${ }^{41}$ Prêter l'oreille aux paroles de leurs chansons peut être source d'enrichissement en ce qu'ils projettent un éclairage sur le Psaume 137.

\section{E ANALYSE COMPARATIVE ET SOCIO-THÉOLOGIQUE}

\section{Le Psaume 137, Babylone et le rastafarisme Ouest Africain}

Bob Marley est sans conteste le promoteur musical du mouvement rastafarien dans le monde à travers la musique reggae. ${ }^{42}$ Apparu en Jamaïque dans les années 1930, le rastafarisme s'est progressivement imposé comme un mouvement religieux, social et politique prônant une lecture et une interprétation proafricaine de la Bible et luttant pour l'émancipation des peuples noirs. ${ }^{43}$ Selon

39 Thomas Römer, Psaumes interdits: du silence à la violence de Dieu (Poliez-leGrand: Éditions du Moulin, 2007), 82.

40 W. VanGemeren, "Psalms," in The Expositor's Bible Commentary: Psalms, Proverbs, Ecclesiastes, Song of Songs (vol. 5; ed. F.E. Gaebelein; Grand Rapids: Zondervan, 1991), 832.

41 Les chantres, poètes et artistes dont nous examinons les paroles des chansons dans ce travail ne se réclament pas chrétiens ni disciples du Christ, ni de près ni de loin. À notre connaissance, les deux artistes dont les œuvres sont citées dans ce travail sont des musulmans. Ce qui nous intéresse, c'est le contenu de leurs messages qui s'adresse à tous leurs mélomanes, toutes religions confondues.

42 Joseph Thompson, "From Judah to Jamaica: The Psalms in Rastafari Reggae," Religion and the Arts 16 (2012): 340-43.

43 Nathaniel S. Murrell, "Introduction: The Rastafari Phenomenon," in Chanting Down Babylon: The Rastafari Reader (ed. N.S. Murrell, W.D. Spencer and A.A. McFarlane; Philadelphia: Temple University Press, 1998), 1-10. Récemment, le 29 Novembre 2018, l'UNESCO a pris la décision d'inscrire le reggae sur la liste du Patrimoine Culturel Mondial témoignant ainsi de l'influence de cette musique à travers le monde. Voir la déclaration sur le site web de l'UNESCO. UNESCO, Culture, 
Murrell, l'attachement des Rastas pour la Bible est dû à une combinaison de facteurs historiques, socioreligieux et culturels. Les références à l'Éthiopie et à l'Afrique ainsi que la ferme conviction que la Bible s'intéresse aux noirs constituent en particulier des centres d'intérêts majeurs. ${ }^{44}$

Les Psaumes sont encore plus appréciés en raison de leur langage poétique et de leur puissance rhétorique. Les rastas trouvent dans les Psaumes et en particulier dans les Psaumes de lamentation, de la matière première en abondance qui se prête à leur idéologie de libération et de lutte pour l'émancipation des noirs. Dans cette lutte, Babylone et Sion sont devenus des lieux symboliques reconfigurés à volonté dans une herméneutique au service de la libération et de l'identité des noirs. Ainsi, Babylone est le symbole du mal, de la corruption politique et économique contre lequel il faut implorer la colère de Dieu. ${ }^{45}$ Babylone symbolise également "le colonialisme, l'esclavage, l'exploitation de l'homme, l'immoralité, l'impureté, la misère, l'oppression, le vice, etc." ${ }^{46}$ À l'opposé, Sion, représente "la délivrance, la terre de liberté, la patrie de tous les Noirs et opprimés, la terre promise, le Paradis." ${ }^{\prime 7}$

Il n'est donc pas étonnant qu'au cœur du rastafarisme l'on trouve le Psaume 137. ${ }^{48}$ En se l'appropriant, et en l'actualisant les rastas marquent leur refus capituler en silence devant l'oppresseur. ${ }^{49}$ Les noirs ont une place de choix dans la doctrine rastafariste. Le Psaume 137 s'adresse en conséquence à leur situation de peuples politiquement et économiquement dominés et exploités. Les rastas croient que le noir est "la réincarnation de l'ancien Israël" et que le temps vient où les noirs dirigeront à nouveau le monde comme au temps de l'Égypte ancienne. ${ }^{50}$

Parti de la Jamaïque le reggae s'est imposé comme une musique de résistance dans laquelle une grande partie de la jeunesse africaine se reconnait. Les chansons reggae s'inspirent des textes bibliques qu'ils traduisent souvent librement, expriment le vécu quotidien et restituent les décors de la vie matérielle, morale et psychologique des opprimés aussi bien que des

Intangible Heritage, Lists, "Reggae Music of Jamaica": https://ich.unesco.org/en/RL/reggae-music-of-jamaica-01398.

44 Nathaniel S. Murrell, "Tuning Hebrew Psalms to reggae Rhythms: Rastas' Revolutionary Lamentations for Social Change," Cross Currents 50 (2000/2001): 526.

45 Ibid., 527-528; Thompson, "From Judah to Jamaica," 331-335.

46 P. Dagri, Le rastafarisme, ses musiques et Bob Marley (Abidjan: Nouvelles Éditions Balafons, 2016), 48.

47 Ibid., 48.

48 Murrell, "Tuning Hebrew Psalms," 529-536.

49 Ibid., 529.

50 Dagri, Le rastafarisme, 44. Thompson, "From Judah to Jamaica," 336-340. 
oppresseurs. ${ }^{51}$ Les chants dans le tableau ci-dessous montrent plusieurs degrés de protestation, de subversion et de résistance. Ils sont écoutés par des milliers de jeunes africains qui “ont si mal." Ils finissent dans le pessimisme et n'offrent en réalité pas d'espoir. Tiken Jah Fakoly le dit clairement dans le refrain d'un autre chant: ${ }^{52}$

Ceci n'est qu'un chant

ceci n'est qu'une chanson

ça changera pas nos vies

mais je chante pour ne pas accepter

je chante pour ne pas accepter

je dis non, je dis non

je dis non, je dis non en chanson

\section{Ça me fait si mal ${ }^{53}$}

Ça me fait si mal

Ça me fait beaucoup pleurer

Ça me fait si mal

Mon cœur a toujours saigné

Les foyers de tensions se multiplient!

Les orphelins se multiplient

Les armes se multiplient

Les guerres s'amplifient

Les morts se multiplient

Et nos pleurs s'amplifient

Nos dettes se multiplient

Notre misère s'amplifie

Les réfugiés se multiplient

Et notre désespoir s'amplifie

Ça me fait si mal (2x)

Ça me fait beaucoup pleurer

Ça me fait si mal

Mon cœur a toujours saigné

Quand je pense qu'aujourd'hui

En Afrique on se détruit

Tandis que les puissances rient

Elles aiment nous voir désunis $\mathrm{Oh}$ !

\section{Y'en a marre ${ }^{55}$}

On en a marre

L'Afrique en a marre marre marre

On en a marre

Le peuple en a marre marre marre

Des journalistes assassinés

Parce que des présidents assassins

Des généraux aux commandes

Des populations opprimées

Des aides aux pays détournées

Des populations affamées

Les fonds du pays dilapidés

Les droits de l'homme ignorés

On en a marre

L'Afrique en a marre marre marre

On en a marre

Le peuple en a marre marre marre

51 Dagri, Le rastafarisme, 67-70.

52 Tiken Jah Fakoly, "Je dis non," African Revolution, Universal Music Division Barclay, 2010.

53 Alpha Blondy, "Ça me fait si mal,” Masada, Blue Note Records, 1992.

55 Tiken Jah Fakoly, "Y'en a marre," Le Caméléon, Universal Music Division Barclay, 2000. 
Ça me fait si mal

Ça me fait beaucoup pleurer

Ça me fait si mal

L'Afrique doit se réveiller

Écartons les fauteurs de trouble

Et ces barrières qui nous entourent

Faisons taire un peu les canons

S'entretuer n'est pas la solution

Ça me fait si mal

Ça me fait beaucoup pleurer

Ça me fait si mal

Mes frères doivent se réveiller

\section{Nazara $^{54}$}

Nazara lémané kognoumalon

Toubaboulou mané kognoumalon

Fôlômôlou tôrôni

An-nou bémalou gnanina eh Allah!

Travail forcé ma fôhankô

Esclavage ma fôhankô

Guerre mondiale ma fôhankô

Travail forcé ma fôhankô

Wobékéni toubaboulégné

Olé toubaboulou mané kognoumalon

An-nouna hobékéla nanzaralou gné

Olé nanzaramasson kané kognoumalon

Ah Allah!

Fôlômôlou tôrôni

An-nou bémalou gnanina eh Allah!

Travail forcé ma fôhankô

Esclavage ma fôhankô

Guerre mondiale ma fôhankô

Travail forcé ma fôhankô

Wobékéni toubaboulégné
Après l'abolition de l'esclavage

Ils ont créé la colonisation

Lorsque l'on a trouvé la solution

Ils ont créé la coopération

Comme on dénonce cette situation

Ils ont créé la mondialisation

Et sans expliquer la mondialisation

C'est Babylone qui nous exploite

On en a marre

L'Afrique en a marre marre marre

On en a marre

Le peuple en a marre marre marre

Faut qu'on arrête de cautionner ça,

La vie de nos frères ne compte pas pour cette bande de vanpayas

Stoppons les guerres, gardons la foi

Faya sur tous les chefs d'état qui nous envoient tuer nos brothers

Ils ne nous respectent pas c'est la même chose pour leurs lois

Ils ne regardent même pas quand le peuple réclame ses droits

Ils ne partagent surtout pas l'argent

c'est pas qu'y en a pas

Ils ne font rien pour nos sisters qui se vendent pour vivre dans ce monde-là

On en a marre

L'Afrique en a marre marre marre

On en a marre

Le peuple en a marre marre marre

Des présidents assassins

On veut plus

\footnotetext{
${ }^{54}$ Tiken Jah Fakoly, “Nazara,” Françafrique, Universal Music Division Barclay, 2002.
} 


\begin{tabular}{|l|l|}
\hline Olé toubaboulou mané kognoumalon & Des généraux aux commandes \\
An-nouna hobékéla nanzaralou gné & On n'en veut plus \\
Olé nanzaramasson kané kognoumalon & Des enfants militaires \\
eh Allah! & $\begin{array}{l}\text { On veut plus } \\
\text { Des orphelins de guerre } \\
\text { On n'en veut plus }\end{array}$ \\
Koni-né ka cacao séné & On en a marre \\
Kalé-bé-na gna cacao ho-san & L'Afrique en a marre marre marre \\
Koni-né ka café séné & On en a marre \\
Kalé-bé-na gna café ho-san & Le peuple en a marre marre marre \\
& L'Afrique en a marre \\
Il parait que ça a été imposé & De toutes ces machinations \\
La mondialisation a été imposée & Mon peuple en a marre \\
Mais imposée en faveur de qui? & De toutes ces manipulations \\
Et Africa est toujours dans la merde! & L'Afrique en a marre \\
Non non! & De toutes ces exploitations \\
Kaï mimiri travail forcé-man & Mon peuple en a marre \\
Kaï mimiri guerre mondiale-man & De toute cette oppression \\
Kaï mimiri esclavage-man & L'Afrique en a marre \\
Kaï mahiné farafinoula & \\
Eh Allah! & \\
&
\end{tabular}

\section{Convergences et divergences entre le Psaume 137 et les chants rastafariens}

A l'exemple du Psaume 137, nous retrouvons dans ces chants des thèmes comme le ressentiment, la frustration, les imprécations, la protestation, la dénonciation et l'imploration.

Dans "ça me fait si mal," le père du reggae ivoirien chante sa consternation face aux conséquences désastreuses des guerres et de l'endettement, la misère et la mort. Contrairement aux chantres du Psaume 137 qui avaient suspendu leurs harpes aux soles, l'artiste ivoirien, lui, prend sa guitare et chante sa peine. Émotionnellement, il y a un rapprochement: d'un côté comme de l'autre, c'est la peine et les pleurs après réflexion sur le sort du peuple. "Mon cœur a toujours saigné" traduit cette douleur vive que l'on ressent face à une situation d'injustice en face de laquelle on éprouve une certaine rage, mais devant laquelle on se trouve impuissant. L'attitude de ceux qui sont considérés comme responsables des malheurs du peuple est comparable: l'indifférence et la raillerie. Pour l'artiste et pour ceux qui fredonnent ce chant, il y a cependant la satisfaction d'avoir dit sa part de vérité devant des oppresseurs et leurs acolytes plus puissants.

"Y'en a marre" est beaucoup plus explicite dans sa critique des oppresseurs. Ce chant exprime le cri de cœur de quelqu'un qui en a assez, qui 
n'en peut plus de voir l'exploitation des pauvres par les plus riches: les dirigeants africains et leurs alliés occidentaux qui conspirent pour affamer, exploiter, opprimer le peuple. "C'est Babylone qui nous exploite." Conformément à la doctrine rasta, Babylone symbolise tout système d'exploitation de l'homme par l'homme. Pour Tiken Jah Fakoly, ce monstre hideux qui se métamorphose pour passer inaperçu et ainsi maintenir son emprise mortifère sur les peuples a été identifié tour à tour comme la traite négrière, le colonialisme, la coopération et la mondialisation. Il est toujours assisté par des valets locaux. Dans son indignation, le peuple a une prise de conscience contre "cette bande de vanpayas." En d'autres termes, il traite les dirigeants et leurs maitres de l'occident de vampires. Il va même jusqu'à demander qu'on ouvre le feu sur les dirigeants, "Faya sur tous les chefs d'état qui nous envoient tuer nos brothers...," une déclaration qui, sous certaines circonstances pourrait être qualifiée d'incitation à la haine et à la violence. La similarité avec le Ps 137.7-9 est frappante. Des paroles aussi violentes ne pourraient être acceptées que lorsque le véhicule qui les transporte est adapté. Les oppresseurs peuvent s'en moquer ou les mépriser, mais ils sont loin de paniquer lorsqu'ils sont traités de vampires ou lorsqu'on ordonne de tirer sur eux. L'arme la plus redoutable de cette chanson ce ne sont pas les paroles violentes, mais la prise de conscience qu'elle prône. La chanson "Nazara" est une réflexion des aspects douloureux du passé de l'Afrique: esclavage, travail forcé, guerre mondiale. C'est une chanson souvenir pleine de mélancolie comme le Ps 137. L'artiste y dénonce l'ingratitude des nazara, c'est-à-dire des blancs face aux souffrances et aux sacrifices des noirs. Dans son désarroi, seul Dieu semble lui offrir une lueur d'espoir d'où l'expression Eh Allah! "Oh, Dieu" souvent caractéristique d'une détresse certaine ou imminente.

L'emploi du "nous" dans les chansons traduit un sentiment d'appartenance à une même communauté de souffrance. C'est un "nous" de solidarité qui rattache celui qui parle aux ancêtres qui ont souffert pendant la traite négrière et pendant la colonisation. De même ce "nous" le rend solidaire de ses frères qui souffrent les effets de la mondialisation et de la coopération. Le premier couplet de 'ça me fait si mal' se termine par "Et notre désespoir s'amplifie." Se référant aux puissances occidentales moqueuses, l'artiste observe qu'"elles aiment nous voir désunis Oh!' Dans 'Y'en a marre', le pronom indéfini “on" représente la communauté des victimes et des mécontents. Plus loin, "la vie de nos frères ne compte pas pour cette bande de vanpayas. Stoppons les guerres, gardons la foi... Ils ne font rien pour nos sisters qui se vendent pour vivre dans ce monde-là." Dans "Nazara," l'artiste raconte les souffrances de "nos ancêtres" dans le passé, an-nou bémalou gnanina eh Allah. En guise de solidarité, il affirme que le travail forcé, l'esclavage et la guerre mondiale ma fôhankô, c'està-dire qu'ils "ne nous ont pas épargné." Ainsi, par l'emploie du "nous," il se crée une chaine Trans générationnelle entre les générations passées et présentes, renforçant ainsi le sentiment d'appartenance à une même communauté de destin marquée par la souffrance. 
Les chansons reggae évoquent ainsi bien des éléments thématiques déjà présents dans le Psaume 137. Elles confirment notre analyse du Psaume 137 comme un Psaume de tous les temps qui reflète les angoisses, les luttes et les espoirs des peuples opprimés.

Il convient d'ajouter que pour l'auteur du Psaume 137 comme pour les rastas africains que nous avons considérés, la motivation première dans la création artistique et poétique n'est pas la haine de l'autre, mais l'amour et la loyauté pour la patrie chez le premier, et pour l'Afrique chez les second. Dans son dernier album paru en mai 2019 et intitulé "le monde est chaud," le reggae man ivoirien Tiken Jah Fakoly exprime ses pensées et ses sentiments sur l'Afrique. Il y chante sa fierté d'être africain et déclare son amour pour ce continent qui a tant souffert, mais qui continue de lutter. Cet extrait du titre, We Love Africa, suffit pour illustrer notre propos :

We love Africa (2), we love

We say, we love Africa (2) we love.

We are Africans, proud to be Africans,

And we are black men, proud to be black men.

When I wake up in the morning and I see the sun shining

I say thank you Grand Daddy because you made me African.

We love Africa (2), we love

We say, we love Africa (2) we love

We have been slaves for 400 hundred years.

When colonization came, it came to the black men.

When I wake up in the morning and I see the sun shining

I say thank you Grand Daddy but we are still fighting. ${ }^{56}$

\section{F CONCLUSION}

Cet article a d'abord cherché à appliquer une herméneutique qui met en valeur l'exégèse biblique et les éléments du contexte africain pour analyser le Psaume 137. En suivant la structure littéraire et thématique du Psaume, en relation avec certains chants dont le genre littéraire se rapproche de la lamentation, nous avons isolé une progression du Psaume qui respecte le texte biblique et qui intègre les aspects sociologiques et émotifs qui donnent naissance à ce type de littérature. En comparant le Psaume 137 à des chants reggae contemporains de la Côte d'Ivoire, nous avons pu mettre en évidence le caractère subversif et revendicatif latent dans le Psaume 137. Les générations successives d'israélites qui ont récité ou chanté ce Psaume trouvent une continuité et un espoir dans le destin qui les lie les unes aux autres. De la même manière, ceux qui frédonnent les chansons engagées des rastas s'identifient aux masses souffrantes du passé et du présent. La parole parfois violente exprimée dans ces chansons comme dans le Psaume 137 est refus, révolte et protestation face à l'injustice extrême. C'est une parole

56 Tiken Jah Fakoly, "We Love Africa,” Le monde est chaud, Wrasse Records, 2019. 
de libération et d'espoir. Le Psaume 137 continuera de déranger certains, mais pour les chanteurs et amateurs de reggae, il demeurera une source intarissable d'inspiration, l'emblème de la lutte entre le mal et le bien.

\section{G BIBLIOGRAPHIE}

Ahn, John. "Psalm 137: Complex Communal Laments." Journal of Biblical Literature 127 (2008): 267-289.

Allen, Leslie C. Psalms 101-150. Word Biblical Commentary 21. Waco: Word Books, 1983.

Alpha Blondy. "Ça me fait si mal." Masada. Blue Note Records, 1992.

Anderson, Arnold Albert. The Book of Psalms. Vol II. Psalms 73-150. New Century Bible Commentary. Grand Rapids: William B. Eerdmans,1972.

Becking, Bob. "Memory and Forgetting in and on Exile: Remarks on Psalm 137." Pages 279-299 in Remembering and Forgetting in Early Second Temple Judah. Edited by Ehud Ben Zvi and Christoph Levin. Tübingen: Mohr Siebeck, 2012.

Bridgeman, Valerie. “'A Long Way from Home': Displacement, Lament, and Singing Protest in Psalm 137." Perspectives in Religious Studies 44 (2017): 213-223.

Briggs, Charles Augustus and Emilie Grace Briggs. The Book of Psalms II. A Critical and Exegetical Commentary. Edinburgh: T \& T Clark, 1907.

Calvin, John. Commentary on the Book of Psalms 93-150. Calvin's Commentaries vol. VI. Translated by James Anderson. Grand Rapids: Baker Book House, 1979.

Dagri, Paul. Le rastafarisme, ses musiques et Bob Marley. Abidjan: Nouvelles Éditions Balafons, 2016.

Dahood, Mitchell. Psalms 101-150. A New Translation with Introduction and Commentary. The Anchor Bible. Garden City, NY: Doubleday, 1970.

Day, John N. Crying for Justice: What the Psalms Teach Us about Mercy and Vengeance in an Age of Terrorism. Grand Rapids: Kregel, 2005.

deClaissé-Walford, Nancy, Rolf A. Jacobson and Neth Laneel Tanner. The Book of Psalms. The New International Commentary on the Old Testament. Grand Rapids: WBEPC, 2014.

Freedman, David N. "The Structure of Psalm 137." Pages 187-205 in Near Eastern Studies in Honor of William Foxwell Albright. Edited Hans Goedicke. Baltimore: John Hopkins University, 1971.

Gaebelein, Grank E. The Expositor's Bible Commentary with the New International Version: Psalms, Proverbs, Ecclesiastes, Songs of Songs. Vol 5. Grand Rapids: Zondervan, 1991.

Gaudet, Frédéric. La Bible annotée. A.T. 6 Psaumes. Saint-Légier: PERLE, 1982.

Gillingham, Susan. "The Reception of Psalm 137 in Jewish and Christian Traditions." Pages 64-82 in Jewish and Christian Approaches to the Psalms. Edited by S. Gillingham. Oxford: Oxford University Press, 2013.

Halle, Morris and J.J. McCarthy. "The Metrical Structure of Psalm 137." Journal of Biblical Literature 50 (1981): 161-167.

Hossfeld, Frank-Lothar and Erich Zenger. Psalms 3: A Commentary on Psalms 101150. Translated by Linda M. Maloney. Minneapolis: Fortress Press, 2011.

Katongole, Emmanuel. Born from Lament: The Theology and Politics of Hope in Africa. Grand Rapids: William B. Eerdmans, 2017.

Kwon, Hyeokil. "Remembrance, Nonidentity, and Lament: A Rereading of Psalm 137 
for Liberation from Unfinished Suffering of Colonization." Korean Christian Theology Publications (2012): 59-78.

Lelièvre, André et Alphonse Maillot. Les Psaumes: Chants d'amour 76-150. Lyon: Olivetant, 2008.

Lenowitz, Harris. “The Mock-Simha of Psalm 137." Pages 149-159 in Directions in Biblical Hebrew Poetry. Edited by E. R. Follis. Journal for the Study of the Old Testament Supplement 90. Sheffield: Sheffield, 1987.

Lombaard, Christo. "Biblical Spirituality, the Psalms, and Identification with the Suffering of the Poor: A Contribution to the Recent African Discussion on Psalm 109." Scriptura 110 (2012): 273-281.

Luc, Alex. "Interpreting the Curses in the Psalms." Journal of the Evangelical Theological Society 42 (1999): 395-410.

Luke, F. "The Songs of Zion as a Literary Category of the Psalter." Indian Journal of Theology 14 (1965): 72-90.

Maré, Leonard P. "Psalm 137: Exile—-Not the Time for Singing the Lord's Song." Old Testament Essays 23 (2010):116-128.

McCann, J. Clinton. "The Hope of the Poor: The Psalms in Worship and Our Search for Justice." Pages 155-178 in Touching the Altar: The Old Testament for Christian Worship. Edited by Carol M. Bechtel. Grand Rapids: William B. Eerdmans, 2008.

Metz, Johann Baptist. Faith in History and Society: Toward a Practical Fundamental Theology. Translated by Matthew Ashley. New York: Cross Publishing, 2007.

Murrell, Nathaniel Samuel. "Introduction: The Rastafari Phenomenon." Pages 1-22 in Chanting Down Babylon: The Rastafari Reader. Edited by N. S. Murrell, W. D. Spencer and A.A. McFarlane. Philadelphia: Temple University Press, 1998.

"Tuning Hebrew Psalms to Reggae Rhythms: Rastas' Revolutionary Lamentations for Social Change.” Cross Currents 50 (2000/2001): 525-540.

Oesterly, W.O.E. The Psalms. London: SPCK, 1955.

Ogden, Graham S. "Prophetic Oracles against Foreign Nations and Psalms of Communal Lament: The Relationship of Psalm 137 to Jeremiah 49:7-22 and Obadiah." Journal for the Study of the Old Testament 24 (1982): 89-97.

Ramantswana, Hulisani. "Song(s) of Struggle: A Decolonial Reading of Psalm 137 in Light of South Africa's Struggle Songs.” Old Testament Essays 32 (2019): 464490.

Römer, Thomas. Psaumes interdits: Du silence à la violence de Dieu. Poliez-le-Grand: Editions du Moulin, 2007.

Sadler, Rodney S. Jr., "Singing a Subversive Song: Psalm 137 and 'Coloured Pompey." Pages 447-457 in The Oxford Handbook of Psalms. Edited by William P. Brown. Oxford: Oxford University Press, 2014.

Savran, George. "How Can We Sing a Song of the Lord? The Strategy of Lament in Psalm 137." Zeitschrift für die Altestamentliche Wissenschaft 112 (2000): 43-58.

Simango, Daniel. “A Comprehensive Reading of Psalm 137." Old Testament Essays 31 (2018): 217-242.

Steenkamp, Yolande. "Violence and Hatred in Psalm 137: The Psalm in Its Ancient Social Context." Verbum et Ecclesia 25/1 (2004): 294-310.

Stowe, David W. "History, Memory, and Forgetting in Psalm 137." Pages 137-158 in The Bible in the Public Square 27. Edited by M. E. Chancey, C. Meyers and C. E. Meyers. Atlanta: SBL Press, 2014 
Song of Exile: The Enduring Mystery of Psalm 137. New York: Oxford University Press, 2016.

Terrein, Samuel. The Psalms: Strophic Structure and Theological Commentary. Grand Rapids: William B. Eerdmans, 2003.

Tiken, Jah Fakoly. "Y’en a marre." Le Caméléon. Universal Music Division Barclay, 2000.

. "Nazara,” Françafrique. Universal Music Division Barclay, 2002.

. "Je dis non.” African Revolution. Universal Music Division Barclay, 2010.

. "Days of Slavery." Racines. Universal Music Division Barclay, 2015.

. "We Love Africa," Le monde est chaud, Wrasse Records, 2019.

Thompson, Joseph. "From Judah to Jamaica: The Psalms in Rastafari Reggae." Religion and the Arts 16 (2012): 328-356.

Verhey, Allen. The Christian Art of Dying: Learning from Jesus. Grand Rapids: William B. Eerdmans, 2011.

Vos, Johannes G. "The Ethical Problem of the Imprecating Psalms." Westminster Theological Journal 4 (1992):123-138.

Weiser, Arthur. The Psalms. The Old Testament Library. Translated by Herbert Hartwell. Philadelphia: The Westminster Press, 1962.

Wénin, André, Psaumes censurés: Quand la prière a des accents violents. Lire la Bible 192. Paris: Editions Cerf, 2017.

Zenger, Erich. A God of Vengeance? Translated by Linda M. Maloney. Louisville: Westminster John Knox, 1996.

Dr. Yacouba Sanon. Theology Department, Université de l'Alliance Chrétienne d'Abidjan. E-mail: ysanon@gmail.com. ORCID: https://orcid.org/0000-0002-2982$\underline{6231}$. 